\title{
Present status of coupled-channels calculations for heavy-ion subbarrier fusion reactions
}

\author{
K. HAGINO ${ }^{1,2,3}$ and J.M. YAO ${ }^{1,4}$
}

${ }^{1}$ Department of Physics, Tohoku University, Sendai 980-8578, Japan

2 Research Center for Electron Photon Science, Tohoku University, Sendai 982-0826, Japan

3 National Astronomical Observatory of Japan, 2-21-1 Osawa, Mitaka, Tokyo 181-8588, Japan

4 School of Physical Science and Technology, Southwest University, Chongqing 400715, China

\begin{abstract}
The coupled-channels method has been a standard tool in analyzing heavy-ion fusion reactions at energies around the Coulomb barrier. We investigate three simplifications usually adopted in the coupledchannels calculations. These are i) the exclusion of non-collective excitations, ii) the assumption of coordinate independent coupling strengths, and iii) the harmonic oscillator approximation for multiphonon excitations. In connection to the last point, we propose a novel microscopic method based on the beyond-mean-field approach in order to take into account the anharmonic effects of collective vibrations.
\end{abstract}

\section{Introduction}

The field of heavy-ion subbarrier fusion reactions started in the late '70s, when a large enhancement of fusion cross sections was experimentally discovered with respect to the prediction of a simple potential model [1]. Even though the potential model works well for light systems, such as ${ }^{14} \mathrm{~N}+{ }^{12} \mathrm{C}$, 
it has turned out that it largely underestimates fusion cross sections for heavier systems, such as ${ }^{16} \mathrm{O}+{ }^{154} \mathrm{Sm}$, at energies below the Coulomb barrier. It has been well recognized by now that this large enhancement of subbarrier fusion cross sections is caused by the couplings of the relative motion between the colliding nuclei to several nuclear intrinsic degrees of freedom, such as low-lying collective excitations in the colliding nuclei as well as several nucleon transfer processes [1-4].

Naturally a standard framework for heavy-ion subbarrier fusion has thus been the coupled-channels method [5] by including relevant degrees of freedom. This method has not only successfully accounted for the subbarrier enhancement of fusion cross sections for many systems but has also provided a natural interpretation of the so called fusion barrier distributions $[1,2]$.

In the coupled-channels approach for heavy-ion fusion reactions, the following simplifications are usually employed. Firstly, the model space is restricted only to low-lying collective excitations, excluding non-collective excitations and giant resonances. Secondly, the coupling strength and the excitation energy for each state are taken to be the same as those in an isolated nucleus and are assumed to be unaltered during the whole process of fusion reaction. Thirdly, a simple harmonic oscillator or a rigid rotor is assumed when multiple excitations to higher collective states are involved. In this contribution, we shall investigate the validity of each of these assumptions.

\section{Role of non-collective excitations}

Let us start with the first assumption, that is, the role of non-collective excitations, which are usually not included in coupled-channels calculations.

Low-lying collective motions are strongly coupled to the ground state, and also have a strong mass number and atomic number dependences. They play a major role in heavy-ion subbarrier fusion reactions, and are explicitly taken into account in coupled-channels calculations. In addition to the lowlying collective excitations, there are many other modes of excitations in atomic nuclei. Among them, non-collective excitations couple only weakly to the ground state and usually they do not affect in a significant way heavy-ion fusion reactions, even though the number of non-collective states is large [6]. Couplings to giant resonances are relatively strong due to their collective character. However, since their excitation energies are relatively large and also are smooth functions of mass number, their effects can be effectively incorporated in a choice of internuclear potential through the 


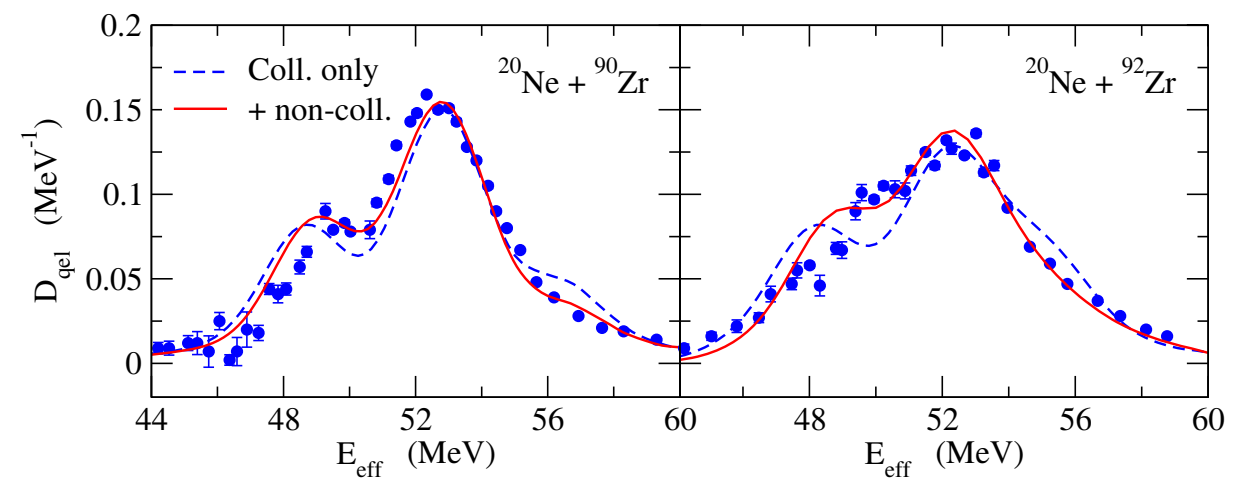

Figure 1: The quasi-elastic barrier distributions for the ${ }^{20} \mathrm{Ne}+{ }^{90} \mathrm{Zr}$ (the left panel) and the ${ }^{20} \mathrm{Ne}+{ }^{92} \mathrm{Zr}$ (the right panel) systems. Here, the quasi-elastic barrier distribution is defined as $D_{\mathrm{qel}}=-d\left[d \sigma_{\mathrm{qel}} / d \sigma_{\mathrm{R}}\right] / d E$, where $\sigma_{\mathrm{qel}}$ and $\sigma_{\mathrm{R}}$ are the quasielastic and the Rutherford cross sections, respectively. These barrier distributions are evaluated at the scattering angle of $\theta_{\text {lab }}=150^{\circ}$ and are plotted as a function of effective energy defined by $E_{\text {eff }}=2 E \sin \left(\theta_{\text {c.m. }} / 2\right) /\left(1+\sin \left(\theta_{\text {c.m. }} / 2\right)\right)$. The dashed lines show the results of the coupled-channels calculations with the collective excitations in the projectile and the target nuclei, while the solid lines take in addition the non-collective excitations in the target nuclei into account with a random matrix model. The experimental data are taken from Ref. [7].

adiabatic potential normalization [1].

Although in most of cases, the non-collective excitations do not play a significant role unless the system is not very heavy, there are certain cases in which they play a role. An example is the ${ }^{20} \mathrm{Ne}+{ }^{92} \mathrm{Zr}$ system, for which the quasi-elastic scattering at backward angles has been measured experimentally [7]. Here, the quasi-elastic scattering refers to the sum of elastic, inelastic, and transfer cross sections, and is a counter part of fusion reactions [8]. Figure 1 shows a comparison between the quasi-elastic barrier distribution, defined as $D_{\mathrm{qel}}=-d\left[d \sigma_{\mathrm{qel}} / d \sigma_{\mathrm{R}}\right] / d E$, where $\sigma_{\mathrm{qel}}$ and $\sigma_{\mathrm{R}}$ are the quasi-elastic and the Rutherford cross sections, respectively, for the ${ }^{20} \mathrm{Ne}$ $+{ }^{90} \mathrm{Zr}$ system and that for the ${ }^{20} \mathrm{Ne}+{ }^{92} \mathrm{Zr}$ systems [9]. One striking feature is that the experimental quasi-elastic barrier distribution for the ${ }^{20} \mathrm{Ne}+{ }^{92} \mathrm{Zr}$ system is much more smeared than that for the ${ }^{20} \mathrm{Ne}+{ }^{90} \mathrm{Zr}$ system [7]. The dashed lines in the figure show the results of the coupledchannels calculations that include the rotational excitations in ${ }^{20} \mathrm{Ne}$ as well as the collective phonon excitations in ${ }^{90,92} \mathrm{Zr}$. This calculation reproduces the experimental data for the ${ }^{20} \mathrm{Ne}+{ }^{90} \mathrm{Zr}$ system but not for the ${ }^{20} \mathrm{Ne}+$ ${ }^{92} \mathrm{Zr}$ system. The solid lines, on the other hand, take into account also the non-collective excitations in ${ }^{90,92} \mathrm{Zr}$ with a random matrix model [9]. One 
can see that the smearing of quasi-elastic barrier distribution for the ${ }^{20} \mathrm{Ne}$ $+{ }^{92} \mathrm{Zr}$ system is now well reproduced by the non-collective excitations of the ${ }^{92} \mathrm{Zr}$ nucleus, whose level density is much larger than that of ${ }^{90} \mathrm{Zr}$ due to the two extra neutrons outside the $N=50$ shell closure.

\section{Coordinate dependent coupling strength and deep subbarrier fusion hindrance}

The coupled-channels approach expands the total wave function with the basis of isolated nuclei. Important inputs for coupled-channels calculations are, together with an internuclear potential, the excitation energy and the coupling strength for each excitations. Usually the experimental data are available for the excitation energy, and the coupling strength can be estimated from a measured electric transition probability [1]. These values are usually employed in coupled-channels calculations assuming that they are not altered during the reaction process. This assumption has been examined recently by Ichikawa and Matsuyanagi [10]. They have carried out randomphase approximation (RPA) calculations with a two-center shell model potential for the ${ }^{16} \mathrm{O}+{ }^{16} \mathrm{O},{ }^{40} \mathrm{Ca}+{ }^{40} \mathrm{Ca}$, and ${ }^{16} \mathrm{O}+{ }^{208} \mathrm{~Pb}$ systems, and have demonstrated that the coupling strengths are indeed constant at large distances but they decrease appreciably in the vicinity of the touching point.

This implies that the assumption of the constant coupling strength is reasonable for fusion reactions at energies around the Coulomb barrier. However, at deep subbarrier energies, the inner turning point is close to or even inner the touching point [11], and it is important to take into account the effect of variation of the coupling strength. Notice that these are the energies at which the deep subbarrier fusion hindrance phenomenon has been observed [4]. In fact, the finding of Ichikawa and Matsuyanagi provides a microscopic justification for the damping factor introduced phenomenologically in the adiabatic model for the deep subbarrier fusion hindrance phenomenon [12].

\section{Semi-microscopic modeling of heavy-ion fusion reactions with a beyond-mean field method}

In heavy-ion fusion reactions at energies around the Coulomb barrier, multiple excitations to higher members of collective states, such as multi-phonon states and high-spin states in the ground state rotational band, often play an 


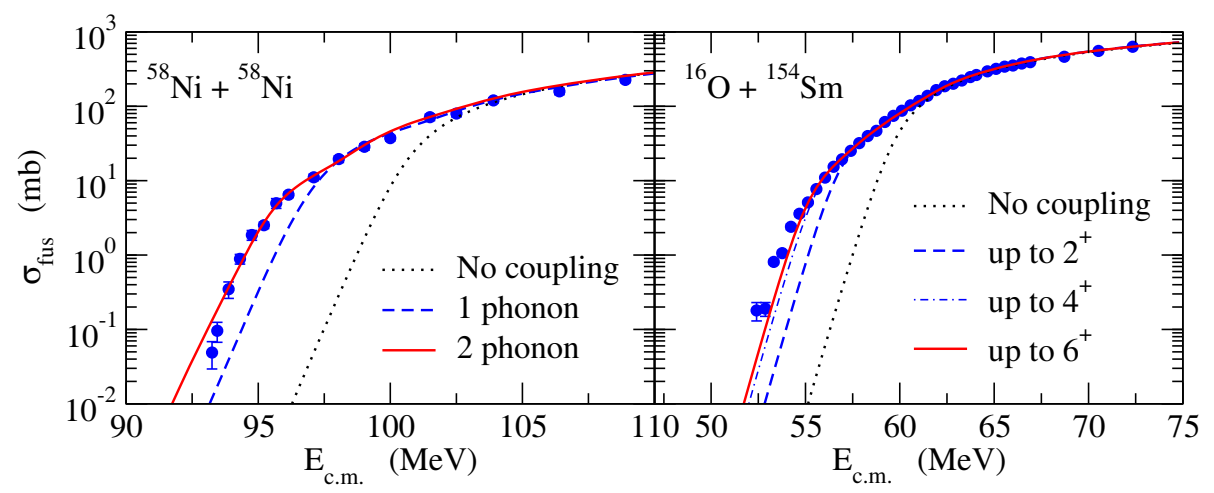

Figure 2: The results of coupled-channels calculations for the fusion cross sections for the ${ }^{58} \mathrm{Ni}+{ }^{58} \mathrm{Ni}$ (the left panel) and the ${ }^{16} \mathrm{O}+{ }^{154} \mathrm{Sm}$ (the right panel) systems. For the ${ }^{58} \mathrm{Ni}+{ }^{58} \mathrm{Ni}$ system, the vibrational coupling to multi-quadrupole-phonon states are considered in the harmonic oscillator approximation. On the other hand, for the ${ }^{16} \mathrm{O}+{ }^{154} \mathrm{Sm}$ system, the rotational coupling within the ground state rotational band is taken into account. The experimental data are taken from Refs. [13,14].

important role. Figure 2 shows typical examples for this. The left and the right panels show fusion cross sections for the ${ }^{58} \mathrm{Ni}+{ }^{58} \mathrm{Ni}$ and the ${ }^{16} \mathrm{O}+{ }^{154} \mathrm{Sm}$ systems obtained with the coupled-channels calculations. For the former system, the vibrational coupling to quadrupole phonon states are considered, while the rotational coupling within the ground state rotational band is taken into account for the latter system. One can see that for both the systems the coupling to the first excited state is insufficient and the couplings to the higher members are necessary in order to account for the subbarrier enhancement of fusion cross sections. This feature has been demonstrated beautifully also through the analyses of fusion barrier distributions $[2,14,15]$.

In order to take into account those multiple excitations in coupledchannels calculations, one usually uses the rigid rotor model for deformed nuclei and the harmonic oscillator model for vibrational nuclei [1]. In reality, however, most nuclei have neither a pure harmonic oscillator spectrum nor a pure rigid body rotational band, although the rigid rotor approximation is reasonable for medium-heavy and heavy deformed nuclei. For example, the ${ }^{58} \mathrm{Ni}$ nucleus, which has usually been considered to be a typical vibrational nucleus, does not exhibit a level spectrum characteristic to the harmonic vibration, e.g., the degeneracy of the two-phonon triplet is considerably broken. Moreover, a recent theoretical calculation also indicates that the $B(E 2)$ strengths among the collective levels in ${ }^{58} \mathrm{Ni}$ deviate largely from the harmonic oscillator limit [16]. 


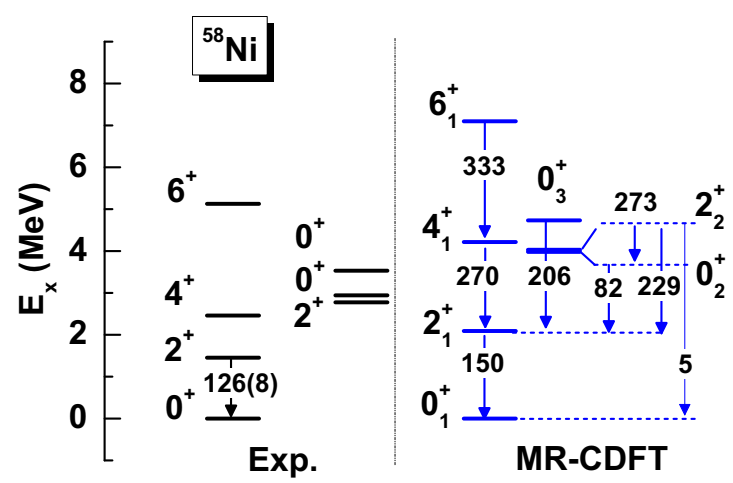

Figure 3: The low-lying energy spectra of ${ }^{58} \mathrm{Ni}$ obtained with the multi-reference covariant density functional theory (MR-CDFT) method with the PC-PK1 force. The arrows indicate the $E 2$ transition strengths, given in units of $e^{2} \mathrm{fm}^{4}$. The experimental data are taken from Refs. [21,22].

There are several theoretical ways to describe an anharmonic vibration. Among them, a multi-reference density-functional theory (MR-DFT) has been rapidly developed for the past decade [17-19]. This method is based on the so called beyond-mean-field approximation, which incorporates the angular momentum and particle number projections as well as the quantum fluctuation of the mean-field wave function described by the generator coordinate method (GCM).

Figure 3 shows the result of the MR-DFT calculation for the ${ }^{58} \mathrm{Ni} \mathrm{nu}-$ cleus. The calculation employs the covariant density functional theory (CDFT) with PC-PK1 interaction [23], and we thus call it MR-CDFT. One can see that the main feature of the energy spectrum, as well as the E2 transition strength from $2_{1}^{+}$to $0_{1}^{+}$, are reproduced rather well. It is interesting to notice that the overall pattern of $B(E 2)$ values is quite different from what would be expected for a harmonic vibrator, even though the excitation energies of the $4_{1}^{+}, 2_{2}^{+}$, and $0_{2}^{+}$states are about twice the energy of the $2_{1}^{+}$ state. In particular, the $E 2$ transition from the $0_{2}^{+}$to the $2_{1}^{+}$states is much smaller than that from the $4_{1}^{+}$and the $2_{2}^{+}$states to the $2_{1}^{+}$state. Instead, the $0_{2}^{+}$state has a strong transition from the $2_{2}^{+}$state, which clearly indicates that the $0_{2}^{+}$state is not a member of the two-phonon triplet. Compared to the $0_{2}^{+}$state, the $E 2$ transition strength from the $0_{3}^{+}$to the $2_{1}^{+}$states is much larger and is comparable to that from the $4_{1}^{+}$and the $2_{2}^{+}$states to the $2_{1}^{+}$state. This fact makes the $0_{3}^{+}$state a better candidate for a member of the two-phonon triplets, even though the excitation energy is a little bit large. 

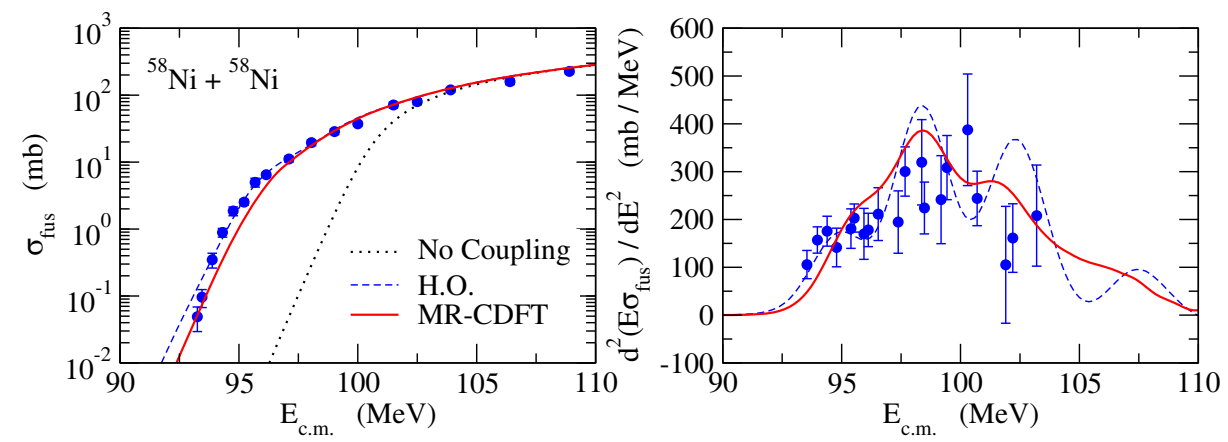

Figure 4: The fusion cross sections (the left panel) and the fusion barrier distributions (the right panel) for the ${ }^{58} \mathrm{Ni}+{ }^{58} \mathrm{Ni}$ system. The dashed line is the result of the coupled-channels calculations including the double quadrupole phonon excitations in each ${ }^{58} \mathrm{Ni}$ nucleus in the harmonic oscillator limit, while the solid line is obtained with the multi-reference covariant density functional theory (MR-CDFT) method by including the $0_{1}^{+}, 2_{1}^{+}, 0_{3}^{+}, 2_{2}^{+}$, and $4_{1}^{+}$states. The dotted line in the left panel denotes the result in the absence of the channel couplings. The experimental data are taken from Ref. [13] for the fusion cross sections and from Ref. [15] for the fusion barrier distribution.

Notice that, in the harmonic oscillator limit, the $B(E 2)$ value from any of the two-phonon triplet states to the $2_{1}^{+}$state is exactly twice the $B(E 2)$ value from the $2_{1}^{+}$state to the ground state. The calculated $B(E 2)$ values shown in Fig. 3, together with the strong transition from the $2_{2}^{+}$to the $\mathrm{O}_{2}^{+}$states, indicate the presence of large anharmonicity in the quadrupole vibrations in ${ }^{58} \mathrm{Ni}$. That is, the calculated $B(E 2)$ values are significantly quenched from the values in the harmonic limit.

We can now ask ourselves how the deviation of the spectrum from the harmonic limit affects the subbarrier fusion reactions of $\mathrm{Ni}$ isotopes. Figure 4 shows the fusion cross section $\sigma_{\text {fus }}(E)$ and the fusion barrier distribution $D_{\text {fus }}(E)=d^{2}\left(E \sigma_{\text {fus }}\right) / d E^{2}[2,24]$ for the ${ }^{58} \mathrm{Ni}+{ }^{58} \mathrm{Ni}$ reaction. The dashed line shows the result of the coupled-channels calculations including up to the double phonon states in the harmonic oscillator limit. All the mutual excitations between the projectile and the target nuclei are included. On the other hand, the solid line in the figure is obtained with the coupling strengths calculated with the MR-CDFT method while adopting a phenomenological Woods-Saxon potential. To this end, we include the $0_{1}^{+}, 2_{1}^{+}, 0_{3}^{+}, 2_{2}^{+}$, and $4_{1}^{+}$ states in the coupled-channels calculations. Again, all the mutual excitation channels are taken into account. See Ref. [20] for the details of the calculations. For a comparison, the figure also shows the result of no-coupling limit by the dotted line. One can see that the calculations in the harmonic limit 
overestimate fusion cross sections at the two lowest energies, while the MRCDFT calculations underpredict fusion cross sections around $95 \mathrm{MeV}$. For the energy dependence of fusion cross sections, shown in terms of fusion barrier distribution in the right panel of the figure, the MR-CDFT calculation leads to a minor improvement by considerably smearing each peak.

We have carried out similar calculations also for the ${ }^{40} \mathrm{Ca}+{ }^{58} \mathrm{Ni}$ system [20]. We have observed again also for this system that the anharmonicity effect in ${ }^{58} \mathrm{Ni}$ smears the fusion barrier distribution, leading to a better agreement with the experimental fusion barrier distribution as compared to the results in the harmonic oscillator limit.

\section{Summary}

The coupled-channels approach has been a standard tool in the field of heavy-ion subbarrier fusion reactions. We have discussed three assumptions used in usual coupled-channels calculations.

We have first discussed the role of non-collective excitations. Usually, coupled-channels calculations take into account a few selected low-lying collective states, neglecting the couplings to non-collective states. We have demonstrated that even though the non-collective excitations can indeed be neglected in many cases, there are certain systems which show those effects. One example is ${ }^{20} \mathrm{Ne}+{ }^{92} \mathrm{Zr}$ system, which shows a considerably smeared barrier distribution as compared to the ${ }^{20} \mathrm{Ne}+{ }^{90} \mathrm{Zr}$ system. By explicitly including many non-collective excitations, we have demonstrated that they indeed smear the barrier distribution for ${ }^{20} \mathrm{Ne}+{ }^{92} \mathrm{Zr}$ system while the effect is much smaller for the ${ }^{20} \mathrm{Ne}+{ }^{90} \mathrm{Zr}$ system.

We have next discussed two important input quantities in coupledchannels calculations, that is, the coupling strength and the excitation energy for each state. In most of coupled-channels calculations, these quantities are assumed to be unchanged during the reaction process and are taken to be constants. The recent RPA calculations have indicated that this is the case at large distances but they decreases considerably around the touching point. We have argued that this leads to important consequences for the deep subbarrier hindrance of fusion cross sections.

We have then proposed a semi-microscopic approach to heavy-ion subbarrier fusion reactions. The basic idea of this approach is to combine a multi-reference density functional theory (MR-DFT) to a coupled-channels calculation. The MR-DFT provides transition strengths among collective states without resorting to the harmonic oscillator model or the rigid rotor 
model. The advantages of this approach include i) deviations from the harmonic limit as well as the rigid rotor limit can be taken into account, ii) it can therefore be applied also to transitional nuclei, which show neither the vibrational nor the rotational characters, and iii) a natural truncation is introduced in the coupling schemes. We have applied this approach to the ${ }^{58} \mathrm{Ni}+{ }^{58} \mathrm{Ni}$ and ${ }^{40} \mathrm{Ca}+{ }^{58} \mathrm{Ni}$ fusion reactions, and have found that the anharmonicities smear the fusion barrier distributions, somewhat improving the agreement with the experimental data.

One of the important current issues in nuclear reaction theory is to develop a microscopic framework starting from the nucleon degree of freedom. It has however been extremely challenging to construct a fully microscopic theory which is applicable to heavy-ion subbarrier fusion reactions, thus to many-particle quantum tunneling. We believe that the semi-microscopic approach presented in this paper provides an important step towards this direction.

\section{Acknowledgements}

We thank N. Rowley, T. Ichikawa, and S. Yusa for useful discussions. This work was partially supported by the National Natural Science Foundation of China under Grant Nos. 11305134, 11105111, and the Fundamental Research Funds for the Central University (XDJK2013C028).

\section{References}

[1] Hagino K. and Takigawa N., Prog. Theor. Phys. 128 (2012) 1001.

[2] Dasgupta M., Hinde D.J., Rowley N. and Stefanini A.M., Annu. Rev. Nucl. Part. Sci. 48 (1998) 401.

[3] Balantekin A.B. and Takigawa N., Rev. Mod. Phys. 70 (1998) 77.

[4] Back B.B., Esbensen H., Jiang C.L. and Rehm K.E., Rev. Mod. Phys. 86 (2014) 317.

[5] Hagino K., Rowley N. and Kruppa A.T., Comp. Phys. Comm. 123 (1999) 143.

[6] Yusa S., Hagino K. and Rowley N., Phys. Rev. C 88 (2013) 044620.

[7] Piasecki E. et al., Phys. Rev. C80 (2009) 054613. 
[8] Hagino K. and Rowley N., Phys. Rev. C69 (2004) 054610.

[9] Yusa S., Hagino K. and Rowley N., Phys. Rev. C 88 (2013) 054621.

[10] Ichikawa T. and Matsuyanagi K., Phys. Rev. C88 (2013) 011602 (R); arXiv: 1506.07963 .

[11] Ichikawa T., Hagino K. and Iwamoto A., Phys. Rev. C75 (2007) 057603; Phys. Rev. C75 (2007) 064612.

[12] Ichikawa T., Hagino K. and Iwamoto A., Phys. Rev. Lett. 103 (2009) 202701.

[13] Beckerman M. et al., Phys. Rev. C23 (1981) 1581.

[14] Leigh J.R. et al., Phys. Rev. C52 (1995) 3151.

[15] Stefanini A.M. et al., Phys. Rev. Lett. 74 (1995) 864.

[16] Yao J.M., Bender M. and Heenen P.-H., Phys. Rev. C91 (2015) 024301.

[17] Bender M., Heenen P.-H. and Reinhard P.-G., Rev. Mod. Phys. 75 (2003) 121.

[18] Yao J.M., Hagino K., Li Z.P., Meng J. and Ring P., Phys. Rev. C89 (2014) 054306.

[19] Vretenar D., the contribution in this conference.

[20] Hagno K. and Yao J.M., Phys. Rev. C91 (2015) 064606.

[21] National Nuclear Data Center, http://www.nndc.bnl.gov/.

[22] Allmond J.M. et al., Phys. Rev. C90 (2014) 034309.

[23] Zhao P.W., Li Z.P., Yao J.M. and Meng J., Phys. Rev. C82 (2010) 054319 .

[24] Rowley N., Satchler G.R. and Stelson P.H., Phys. Lett. B254 (1991) 25. 